\title{
Estudo normativo do eletrorretinograma por padrões (PERG) em adultos
}

\author{
Pattern-reversal electroretinogram (PERG): a normative study in adults
}

\author{
Letícia Dourado Alves ${ }^{1}$ \\ Adriana Berezovsky² \\ Paula Yuri Sacai ${ }^{3}$ \\ Josenilson Martins Pereira ${ }^{4}$ \\ Solange Rios Salomão ${ }^{5}$
}

\begin{tabular}{|c|}
\hline RESUMO \\
\hline 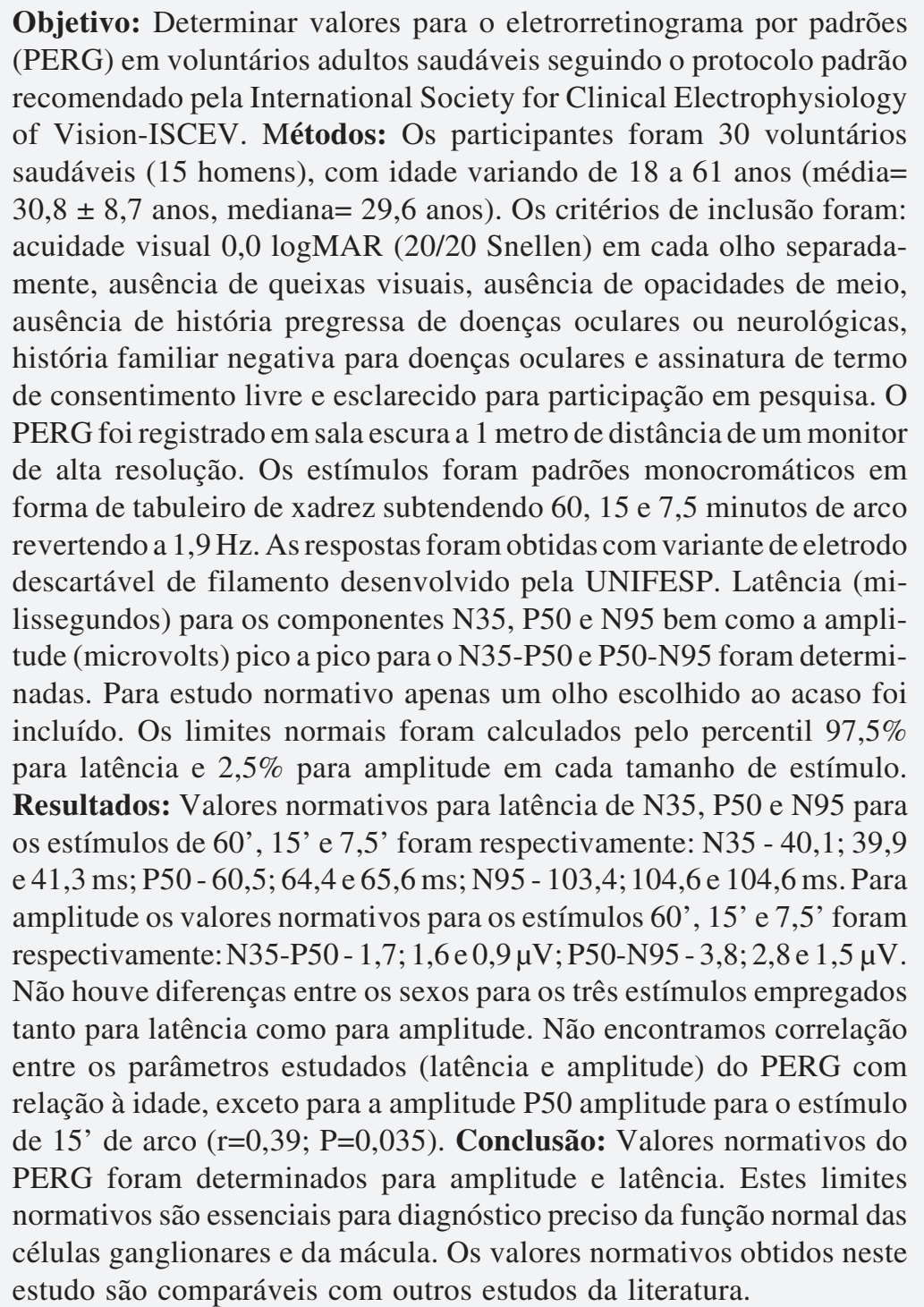 \\
\hline
\end{tabular}

Descritores: Valores de referência; Eletrorretinografia/normas; Eletrofisiologia; Células ganglionares da retina
Trabalho realizado no Departamento de Oftalmologia da Universidade Federal de São Paulo - UNIFESP - São Paulo (SP) - Brasil.

${ }^{1}$ Estagiária do Departamento de Oftalmologia da Universidade Federal de São Paulo - UNIFESP - São Paulo (SP) - Brasil.

${ }^{2}$ Professora Adjunto do Departamento de Oftalmologia da UNIFESP - São Paulo (SP) - Brasil.

${ }^{3}$ Pós-graduanda em Ciências Visuais pela UNIFESP - São Paulo (SP) - Brasil.

${ }^{4}$ Mestre em Ciências Visuais pela UNIFESP - São Paulo (SP) - Brasil.

${ }^{5}$ Livre Docente, Professora Associado do Departamento de Oftalmologia da UNIFESP - São Paulo (SP) - Brasil.

Endereço para correspondência: Solange Rios Salomão. Departamento de Oftalmologia da Universidade Federal de São Paulo. Rua Botucatu, 822 - Vila Clementino - São Paulo (SP) - CEP 04023-062

E-mail: ssalomao@oftalmo.epm.br

Recebido para publicação em 24.09.2009

Última versão recebida em 23.03.2010

Aprovação em 12.04.2010

Nota Editorial: Depois de concluída a análise do artigo sob sigilo editorial e com a anuência do Dr. Pedro Carricondo sobre a divulgação de seu nome como revisor, agradecemos sua participação neste processo. 


\section{INTRODUCÃO}

O eletrorretinograma por padrões (PERG) é uma resposta eletrofisiológica obtida por estimulação da retina central devido a mudanças no contraste de estímulos estruturados (tabuleiros de xadrez ou grades pretas e brancas) com luminância constante ${ }^{(1-4)}$. O PERG é uma ferramenta clínica diagnóstica que requer fixação central, permitindo a avaliação objetiva da função macular e também das células ganglionares da retina $^{(5)}$. As principais indicações deste exame são as disfunções maculares (distrofias maculares, envolvimento macular em doenças retinianas como retinose pigmentária, distrofia cone-bastonete), as doenças do nervo óptico (doenças desmielinizantes, compressão do nervo óptico, atrofia óptica) e glaucoma. É útil também em casos de baixa visual não orgânica, como simulação de perda da visão ${ }^{(1-4)}$ e nos casos de rastreamento para os hipertensos oculares. Tem sido usado tanto na prática clínica como em pesquisa, com utilidade nas áreas de oftalmologia e neurologia ${ }^{(3)}$.

Os elementos retinianos que dão origem a esta resposta ainda são motivo de investigação e sugerem múltipla origem $^{(3)}$. Inicialmente em 1981, a origem do PERG nas células ganglionares da retina foi confirmada pela observação em um estudo eletrofisiológico em que após quatro meses de uma ressecção cirúrgica do nervo óptico de felinos o PERG estava abolido e o eletrorretinograma de campo total (ERG) permaneceu inalterado ${ }^{(6)}$. Porém outros trabalhos mostraram a persistência do P50 mesmo com dano severo ao nervo óptico como ausência de percepção luminosa, criando assim a teoria de que além das células ganglionares haveria outras células geradoras do PERG para este componente, provavelmente células da retina interna, mais distais que as ganglionares ${ }^{(7-8)}$.

Desta maneira os componentes P50 e N95 do PERG possuem uma origem estrutural distinta permitindo que seja seletivamente afetado nas doenças da mácula e do nervo ópti$\mathrm{co}^{(3,5-11)}$. Este fato demonstra a importância do PERG tanto em oftalmologia quanto em neurologia.

O PERG pode apresentar dois tipos de traçados diferentes dependendo da frequência temporal apresentada na estimulação (uma resposta completa antes da próxima reversão do contraste), sendo chamado de "transiente" quando a taxa de reversão temporal dos padrões é de até 6 ciclos por segundo e de estado estável acima de 6 ciclos por segundo ${ }^{(12)}$. O PERG transiente em indivíduos normais consiste em uma onda de baixo sinal composta por três elementos assim distribuídos (Figura 1): um pequeno pico negativo inicial (N35) seguido de um componente positivo maior ao redor de 45-60 ms (P50), que por sua vez é seguido de um componente negativo ao redor de 90-100 ms (N95). A nomenclatura utilizada é baseada nas normas práticas da clínica neurofisiológica pelas quais os componentes são identificados pela polaridade e latência aproximada $^{(4,12-13)}$. Em muitos indivíduos o vale N35 não é definido, sendo substituído pela média entre o tempo zero e $\mathrm{P} 50^{(2-3,9)}$. A sua origem ainda não está estabelecida ${ }^{(1,4,9)}$.

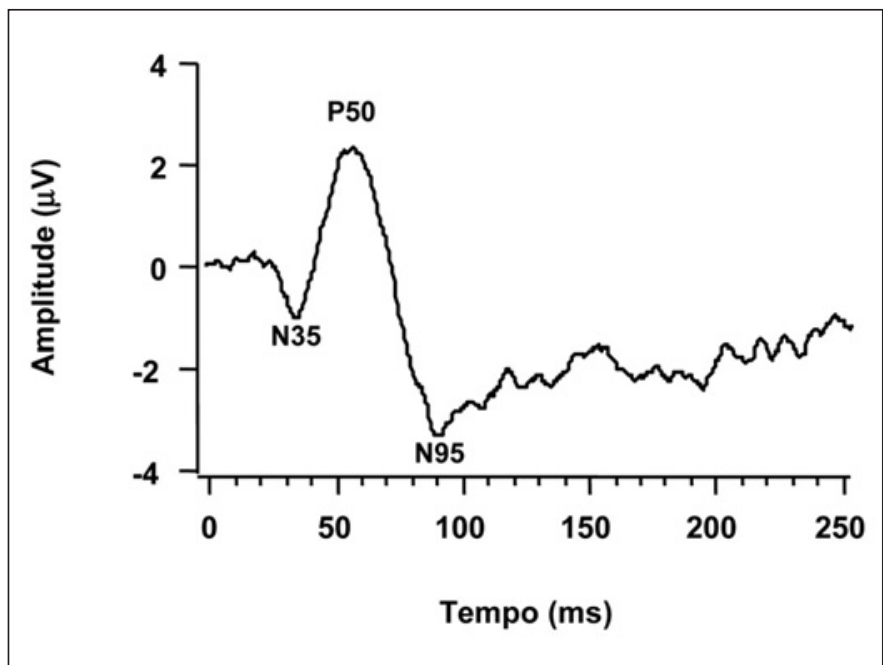

Figura 1 - Registro do eletrorretinograma por reversão de padrões (PERG) obtido de voluntário saudável normal no Laboratório de Eletrofisiologia Visual Clínica da UNIFESP. Nota-se os componentes N35, P50 e N95.

A International Society for Clinical Electrophysiology of Vision (ISCEV) em sua última revisão de padronização do PERG relata que devido à ausência de padrões internacionais de referência, cada laboratório deve determinar seus próprios dados normativos para o estabelecimento de parâmetros mínimos para aquisição, interpretação e posterior comparação de exames eletrofisiológicos realizados por diferentes instituições no mundo ${ }^{(12)}$. A proposta deste estudo é determinar valores normativos para o PERG em voluntários adultos saudáveis seguindo o protocolo padrão recomendado pela ISCEV ${ }^{(12-13)}$.

\section{MÉTODOS}

\section{Participantes}

Foram incluídos neste estudo 30 voluntários saudáveis (15 homens), com idade variando de 18 a 61 anos (média= $30,8 \pm 8,7$ anos, mediana= 29,6 anos), sendo 24 voluntários de cor de pele branca, três voluntários de cor de pele amarela e três de cor de pele negra. Todos os participantes foram informados sobre os procedimentos aos quais seriam submetidos, seus riscos e benefícios. O protocolo de estudo foi aprovado pelo Comitê de Ética em Pesquisa da Universidade Federal de São Paulo (CEP 1174/06) e seguiu os termos da Declaração de Helsinque.

\section{Critérios de Inclusão}

Os critérios de inclusão foram: acuidade visual 0,0 $\log$ MAR (20/20 Snellen) medida em cada olho separadamente, com a melhor correção óptica, utilizando a tabela de optotipos ETDRS retroiluminada, apresentada à distância de quatro metros; ausência de queixas visuais; ausência de 
opacidades de meio; ausência de história pregressa de doenças oculares (ex.: glaucoma, neuropatias e retinopatias) ou neurológicas; ausência de doença sistêmica que pudesse interferir no resultado (ex.: diabetes, hipertensão arterial sistêmica, cefaléia crônica sem esclarecimento); história familiar negativa para doenças oculares (ex.: glaucoma e doenças hereditárias retinianas e do nervo óptico); erro refracional menor que 4 dioptrias (equivalente esférico) e assinatura de termo de consentimento livre e esclarecido para participação em pesquisa.

\section{Registro do PERG}

O registro do PERG foi realizado em sala escura em condição monocular de estimulação, com o primeiro olho escolhido aleatoriamente, com pupila natural para preservar a acomodação e manter a qualidade da imagem retiniana e quando necessário a correção óptica adequada. Cada participante, durante o exame, permaneceu confortavelmente sentado, com um monitor de vídeo de alta resolução com luminância constante ao nível dos olhos no qual foram gerados os padrões, posicionado à distância de 1 metro. Foi solicitada fixação no centro da tela do monitor e durante a realização monitoravase constantemente a fixação, a condição de alerta e o piscar do indivíduo.

O PERG foi captado por uma variante de eletrodo descartável de filamento desenvolvida na UNIFESP (Figura 2). Nesta variante, não há o eletrodo de referência acoplado. Os terminais do eletrodo descartável foram fixados nos cantos lateral e medial de cada olho após limpeza da pele com gel abrasivo (Nuprep ${ }^{\circledR}$ ). O filamento foi inserido no fundo de saco conjuntival inferior permanecendo, após sua colocação, próximo ao limbo ${ }^{(14)}$.

O eletrodo de referência consistiu de um eletrodo de cúpula de ouro, posicionado no canto externo ipsilateral, preenchido com pasta condutora e fixado com fita adesiva. $\mathrm{O}$ eletrodo terra consistiu de eletrodo de cúpula de ouro, posicionado no lobo da orelha ipsilateral, preenchido com pasta condutora e fixado por clipe. Não foram utilizados anestésicos locais ${ }^{(12-13)}$.

A tela do estimulador compreendia $17^{\circ} \times 17^{\circ}$ do campo visual e o estímulo gerado compreendia padrões do tipo tabuleiro de xadrez em preto e branco com estímulos formando ângulos visuais de 60, 15 e 7,5 minutos de arco. A frequência temporal de reversão dos padrões foi de $1,9 \mathrm{~Hz}$. Os contrastes foram utilizados ao máximo (100\%) e a iluminação foi mantida constante. As respostas foram registradas pelo sistema LKC UTAS E-3000 (LKC Technologies Inc., Gaithesburg, MD, USA). O sinal biológico foi amplificado na ordem de 40.000 vezes e filtrado com banda de passagem passa-alto em $100 \mathrm{~Hz}$ e passa-baixo em $0,3 \mathrm{~Hz}$. Foi analisada a resposta média de 100 apresentações do padrão reverso livres de artefatos (traçados não compatíveis). O tempo de análise foi de $250 \mathrm{~ms}$. A impedância foi mantida igual ou inferior a $5 \mathrm{~K}$ Ohms e todos os registros foram obtidos pelo mesmo examinador (LDA).
Ao término do exame todos os sujeitos foram avaliados com luz azul de cobalto após instilação de uma gota de colírio de fluoresceína para evidenciar possível abrasão corneana que pudesse ter sido causada pelo eletrodo, sendo orientados a retornar ao serviço caso houvesse dor, hiperemia ou outro sintoma e/ou sinal ocular.

\section{Parâmetros}

As amplitudes do componente negativo (N35), do pico positivo (P50) e do componente negativo (N95) foram determinadas. Foram analisados a latência (ms) para os componentes N35, P50 e N95 assim como as amplitudes pico a pico N35-P50 e P50-N95 $(\mu \mathrm{V})$.

\section{Método estatístico}

Para cálculo dos dados normativos foram utilizados resultados apenas de um olho escolhido ao acaso.

Os limites normais inferiores foram determinados pelo percentil 97,5\% para os parâmetros de latência e do percentil 2,5\% para amplitude dos três tamanhos de estímulo empregados. O teste t de Student foi feito para análise dos parâmetros do PERG para sexo. Quando o teste de normalidade falhou, foi utilizado o teste de Mann-Whitney. O teste de correlação de Pearson foi utilizado para análise dos parâmetros do PERG com idade.

\section{RESULTADOS}

Os valores normativos para latência (ms) e amplitude $(\mu \mathrm{V})$ para os três tamanhos de estímulo empregados (60', 15' e $\left.7,5^{\prime}\right)$ são mostrados na tabela 1 . A análise estatística não mostrou quaisquer diferenças entre os sexos para os três estímulos empregados tanto para latência como para amplitude.

Os resultados da correlação de Pearson para amplitude e latência do PERG para os três estímulos apresentados em relação a idade são mostrados na tabela 2. Não houve correlação entre nenhum dos parâmetros estudados e a idade dos participantes, exceto fraca correlação positiva entre amplitude P50 para o estimulo de 15 minutos de arco ( $\mathrm{r}=0,39 ; \mathrm{P}=0,035)$.

Apenas um participante queixou-se de olho vermelho após 24 horas do registro do PERG, com exame de biomicroscopia sem alterações, sendo orientado uso de colírio lubrificante e retorno caso persistisse o sinal.

\section{DISCUSS ÃO}

Os resultados de padronização do PERG transiente em voluntários saudáveis obtidos no presente estudo são comparáveis a resultados de estudos anteriores ${ }^{(15-17)}$. Os valores médios de amplitude e latência do PERG transiente determinados em nosso estudo na faixa etária de 18 a 61 anos foram semelhantes a estudo de 20 adultos de 24 a $39 \operatorname{anos}^{(15)}$ e aos valores recentemente descritos em estudo brasileiro com 26 adultos de 18 a $75 \operatorname{anos}^{(16)}$. Provavelmente a semelhança en- 


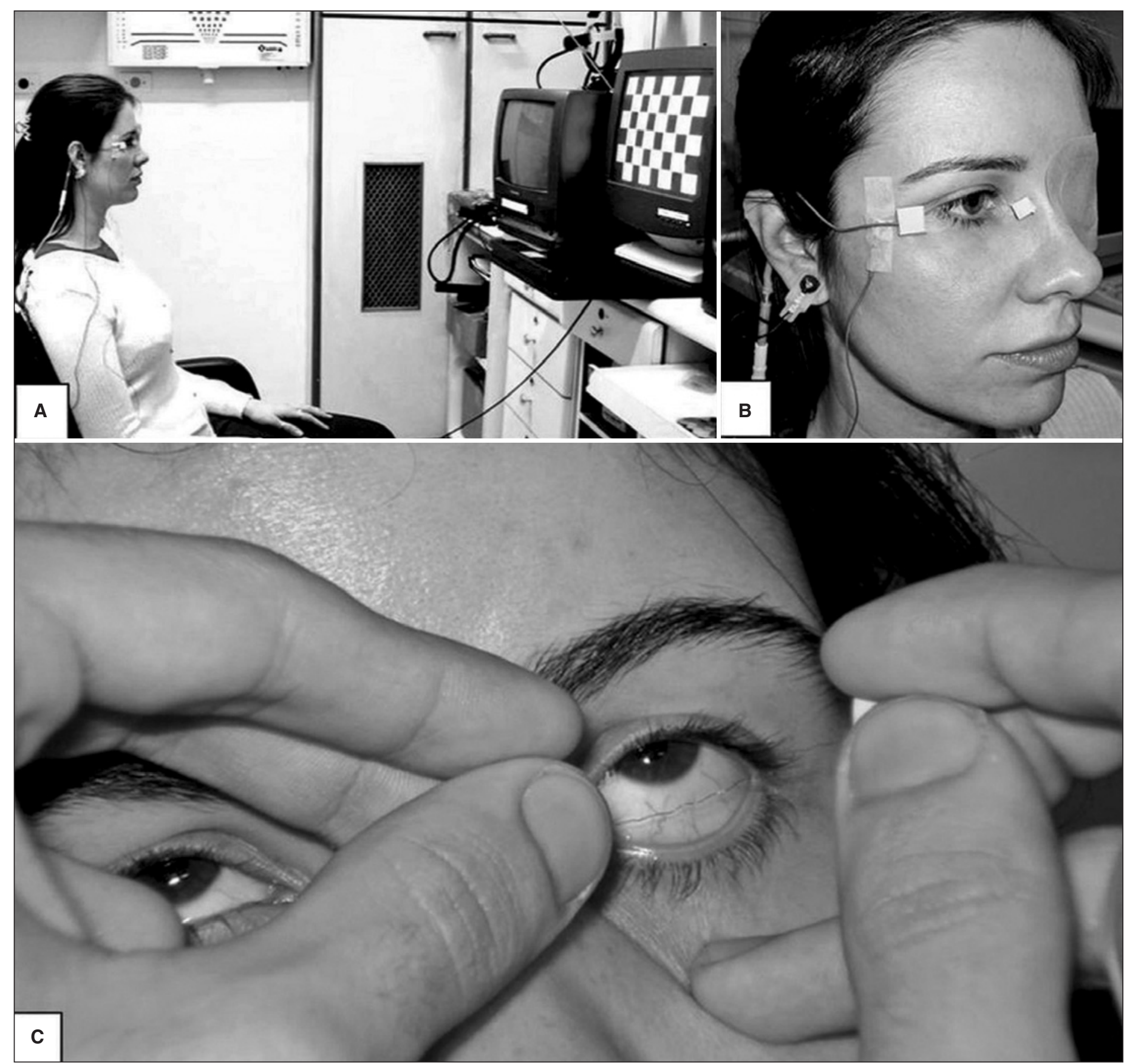

Figura 2 - A) Situação de teste do PERG; B) Posicionamento dos eletrodos; C) Detalhe do eletrodo de fibra UNIFESP

contrada deve-se ao fato de que nestes estudos foi empregada metodologia similar, com registro do PERG por meio de eletrodos de contato, que captam respostas menores, sendo de fácil utilização e não provocam danos oculares.

Como demonstrado em muitos trabalhos o vale N35 não é definido, então é substituído pela média entre o tempo zero e P50 ${ }^{(2-3,9)}$, porém em nosso trabalho foi determinado em todos os participantes isso provavelmente se deve ao fato da qualidade de registro dos exames, ao eletrodo utilizado e também ao fato dos participantes escolhidos serem saudáveis.
Outro fator que dificulta a comparação entre os estudos normativos do PERG é que o PERG se subdivide em transiente (até 6 ciclos por segundos) e estado estável. Um estudo brasileiro padronizou o PERG de estado estável em 48 voluntários saudáveis, porém a diferença no tipo de estimulação não permitiu comparação direta com nossos dados ${ }^{(18)}$. Mesmo que o exame seja realizado dentro das recomendações da ISCEV, deve-se considerar que há fontes de variabilidade como o uso de diferentes tipos de eletrodos de contato $^{(15-18)}$. As fontes de variabilidade que podem influenciar os parâme- 
tros do PERG em estudos normativos são o tamanho da banda de rejeição, o número de apresentações, o posicionamento do eletrodo de referência, o posicionamento e o tipo de eletrodo ativo. A recomendação da ISCEV é que o PERG seja feito de forma transiente, com utilização de eletrodos de filamento ou de folha de ouro, pois permitem melhor posicionamento e não provocam borramento da imagem ${ }^{(12-13)}$. Além do adequado posicionamento do eletrodo ativo e referência serem fundamentais para registros confiáveis e reprodutíveis ${ }^{(8,12,17)}$.

Devido à sua pequena amplitude o registro do PERG é mais difícil do que o de outras respostas eletrofisiológicas e exige grande atenção e experiência do examinador para obtenção de reprodutibilidade, já que o simples ato de piscar pode interferir na qualidade das respostas e gerar artefatos fisiológicos.

Em relação às características raciais da amostra, tentamos aproximar dos últimos dados do IBGE (Instituto Brasileiro de

\begin{tabular}{|c|c|c|c|}
\hline & $60^{\prime}$ & $15^{\prime}$ & $7,5^{\prime}$ \\
\hline Latência de N35 (ms) & 35,1 & 35,1 & 35,8 \\
\hline DP & 2,9 & 2,6 & 2,7 \\
\hline Média +2DP & 40,9 & 40,3 & 41,2 \\
\hline Mediana & 35,0 & 35,0 & 35,0 \\
\hline Percentil $97,5 \%$ & 40,1 & 39,9 & 41,3 \\
\hline Latência de P50 (ms) & 55,6 & 58,4 & 58,4 \\
\hline DP & 3,3 & 3,3 & 4,0 \\
\hline Média +2DP & 62,2 & 65,0 & 66,4 \\
\hline Mediana & 55,0 & 59,0 & 58,5 \\
\hline Percentil $97,5 \%$ & 60,5 & 64,4 & 65,6 \\
\hline Latência de N95 (ms) & 96,5 & 97,8 & 99,0 \\
\hline DP & 3,4 & 3,6 & 3,4 \\
\hline Média +2DP & 103,3 & 105,0 & 105,8 \\
\hline Mediana & 96,2 & 98,2 & 99,5 \\
\hline Percentil $97,5 \%$ & 103,4 & 104,6 & 104,6 \\
\hline Amplitude N35-P50 $(\mu \mathrm{V})$ & 3,3 & 2,7 & 2,1 \\
\hline DP & 1,3 & 0,9 & 0,8 \\
\hline Média - 2DP & 0,7 & 0,9 & 0,5 \\
\hline Mediana & 3,2 & 2,6 & 2,1 \\
\hline Percentil 2,5\% & 1,6 & 1,5 & 0,9 \\
\hline Amplitude P50-N95 $(\mu \mathrm{V})$ & 5,4 & 4,5 & 3,6 \\
\hline DP & 1,2 & 1,0 & 1,2 \\
\hline Média - 2DP & 3,0 & 2,5 & 1,2 \\
\hline Mediana & 5,3 & 4,5 & 3,5 \\
\hline Percentil 2,5\% & 3,8 & 2,8 & 1,5 \\
\hline
\end{tabular}

Geografia e Estatística) de 2000 nos quais na cidade de São Paulo consta cerca de $68 \%$ de Caucasianos, $30 \%$ de Negros e $2 \%$ Amarelos $^{(19)}$. Assim, de acordo com a classificação adotada pelo IBGE desde 1991, a raça Negra é o conjunto de Pardos e Pretos e significa a cor de pele que o indivíduo se autodeclara (quesito cor é subjetivo nos questionários) ${ }^{(19-20)}$. Então, como a classificação racial é arbitrária e não utiliza o conceito biológico e sim étnico, ocorre grande dificuldade para definir a raça do indivíduo em nosso país pela miscigenação social, biológica e cultural.

A recomendação feita pela ISCEV de padronização dos valores de normalidade e também da forma de realização do exame de eletrorretinograma por reversão de padrões é de grande importância na prática diária de um laboratório de eletrofisiologia visual ${ }^{(12-13)}$. Dados normativos obtidos em outro laboratório por vários motivos não podem ser utilizados como parâmetros de normalidade já que raramente os laboratórios de eletrofisiologia usam exatamente o mesmo equipamento para aquisição das respostas, havendo configurações, marcas e modelos diferentes ${ }^{(18)}$. Ocorrem também diferenças nos eletrodos utilizados ${ }^{(14)}$, bem como diferenças raciais da amostra estudada e também da experiência da equipe em realizar o exame justificando assim necessidade de dados normativos próprios tanto para o PERG como para outros exames eletrofisiológicos ${ }^{(21-26)}$.

\section{CONCLUSÃO}

Foram determinados valores normativos para o registro clínico do PERG. Estes parâmetros são fundamentais para o diagnóstico preciso do funcionamento das células ganglionares da retina e da mácula e são comparáveis a estudos anteriores.

\section{ABSTR ACT}

Purpose: To determine normative values for pattern-reversal electroretinogram (PERG) in healthy adult volunteers according to the standard protocol recommended by the International Society for Clinical Electrophysiology of VisionISCEV. Methods: Participants were 30 healthy volunteers (15 males) with ages from 18 to 61 years (mean $=30.8 \pm 8.7$ yrs.; median $=29.5 \mathrm{yrs}$ ). Inclusion criteria were: visual acuity of 0.0 logMAR (20/20 Snellen) in each eye, absence of visual

\begin{tabular}{|c|c|c|c|c|c|c|}
\hline \multirow[t]{2}{*}{ Parâmetro } & \multicolumn{2}{|c|}{$60^{\prime}$} & \multicolumn{2}{|c|}{$15^{\prime}$} & \multicolumn{2}{|c|}{$7,5^{\prime}$} \\
\hline & $r$ & $\mathbf{P}$ & $r$ & $P$ & $r$ & $\mathbf{P}$ \\
\hline Amplitude N35-P50 & 0,105 & 0,582 & 0,3860 & 0,0346 & 0,221 & 0,240 \\
\hline Amplitude P50-N95 & $-0,084$ & 0,658 & 0,3370 & 0,0680 & $-0,228$ & 0,225 \\
\hline Latência P50 & $-0,196$ & 0,300 & $-0,1050$ & 0,5800 & $-0,270$ & 0,149 \\
\hline Latência N95 & $-0,271$ & 0,147 & 0,0126 & 0,9470 & 0,008 & 0,966 \\
\hline
\end{tabular}


complaints, absence of media opacities, negative history for ocular or neurological diseases, negative family history for ocular and informed consent. PERG was recorded from each eye in a darkened room at $1 \mathrm{~m}$ from a high resolution display monitor. Stimuli were monochromatic checkerboards subtending 60, 15 e 7.5 minutes of visual angle reversing at $1.9 \mathrm{~Hz}$. Responses were obtained from modified disposable fiber electrodes developed at UNIFESP. Latency (ms) for N35, P50 and N95 components as well as peak-to-peak amplitudes $(\mu \mathrm{V})$ for N35-P50 and P50-N95 were determined. For normative values only one randomly chosen eye was included. Normal limits were calculated as $97.5 \%$ percentiles for latency and $2.5 \%$ percentile for amplitudes for each stimulus size. Results: Normal limits for N35, P50 and N95 latencies for 60', 15' and 7.5' stimuli were respectively: N35 - 40.1; 39.9 and $41.3 \mathrm{~ms}$; P50 - 60.5; 64.4 and $65.6 \mathrm{~ms}$ and N95 - 103.4; 104.6 and $104.6 \mathrm{~ms}$. For amplitude the normative values for N35-P50 and P50-N95 for 60', 15' and 7.5' were respectively: N35-P50 $1.7 ; 1.6$ and $0.9 \mu \mathrm{V}$; P50-N95 - 3.8; 2.8 and $1.5 \mu \mathrm{V}$. No gender differences were found either for latency or for amplitude in the three stimulus sizes. There was no correlation between PERG latency and amplitude with age, except for P50 amplitude for stimulus $15^{\prime}(\mathrm{r}=0.39 ; \mathrm{P}=0.035)$. Conclusions: Normative values were determined for PERG parameters of amplitude and latency for three stimulus sizes. These parameters are important for evaluating the normal functioning of retinal ganglion cells and the macula. The normative values obtained in this study are comparable to previous studies in the literature.

Keywords: Reference values; Electroretinography/standards; Electrophysiology; Retinal ganglion cells

\section{REFERÊNCIAS}

1. Dantas AM. Eletrorretinografia. In: Dantas AM, Costa JGC, Pacini Neto L, Yamane R, Elias CA. Eletrofisiologia ocular. Rio de Janeiro: Cultura Médica; 1995. p.172-4. (Biblioteca Brasileira de Oftalmologia).

2. Holder GE. The pattern electroretinogram. In: Fishman GA, Birch DG, Holder GE, Brigell MG. Electrophysiologic testing in disorders of the retina, optic nerve, and visual pathway. $2^{\text {nd }}$ ed. San Francisco, CA: The Foundation of the American Academy of Ophthalmology; 200. p.197-235.

3. Holder GE. Pattern electroretinography (PERG) and an integrated approach to visual pathway diagnosis. Prog Retin Eye Res. 2001;20(4):531-61.

4. Berezovsky A, Salomão SR. Eletrorretinografia (ERG) In: Hofling Lima HA, Freitas D, Moeller CTA, Martins EN. Manual de condutas em oftalmologia. UNIFESP - Instituto da Visão. São Paulo: Atheneu; 2008. p.1154-5.
5. Holder GE. Significance of abnormal pattern electroretinography in anterior visual pathway dysfunction. Br J Ophthalmol. 1987;71(3):166-71.

6. Maffei L, Fiorentini A. Electroretinographic responses to alternating gratings before and after section of the optic nerve. Science. 1981;211(4485):953-5.

7. Harrison JM, O'Connor PS, Young RS, Kincaid M, Bentley R. The pattern ERG in man following surgical resection of the optic nerve. Invest Ophthalmol Vis Sci. 1987;28(3):492-9.

8. Sherman J. Simultaneous pattern-reversal electroretinograms and visual evoked potentials in diseases of the macula and optic nerve. Ann N Y Acad Sci. 1982;388:214-26.

9. Holder GE. The pattern electroretinogram in anterior visual pathway dysfunction and its relationship to the pattern visual evoked potential: a personal clinical review of 743 eyes. Eye (Lond). 1997;11(Pt 6):924-34.

10. Zrenner E. The physiological basis of the pattern electroretinogram. Prog Retin Res. 1990;9:427-64.

11. Maffei L, Fiorentini A, Bisti S, Holländer H. Pattern ERG in the monkey after section of the optic nerve. Exp Brain Res. 1985;59(2):423-5.

12. Holder GE, Brigell MG, Hawlina M, Meigen T, Vaegan, Bach M; International Society for Clinical Electrophysiology of Vision. ISCEV standard for clinical pattern electroretinography-2007 update. Doc Ophthalmol. 2007;114(3):111-6.

13. Bach M, Hawlina M, Holder GE, Marmor MF, Meigen T, Vaegan, Miyake Y. Standard for pattern electroretinography. International Society for Clinical Electrophysiology of Vision. Doc Ophthalmol. 2000;101(1):11-8.

14. Berezovsky A, Pereira JM, Salomão SR, Santos VR, Schor P. Validation of a new fiber electrode prototype for clinical electroretinography. Arq Bras Oftalmol. 2008;71(3):316-20.

15. Jacobi CP, Walter P, Brunner R, Krieglstein GK. Reproducibility and intraindividual variability of the pattern electroretinogram. German J Ophthalmol. 1994:3(4-5):216-9.

16. Cunha LP, Oyamada MK, Monteiro ML. Pattern electroretinograms for the detection of neural loss in patients with permanent temporal visual field defect from chiasmal compression. Doc Ophthalmol. 2008;117(3):223-32.

17. Odom VJ, Holder GE, Feghali JG, Cavender S. Pattern electroretinogram intrasession reliability: a two center comparison. Clin Vision Sci. 1992;7(4):263-81.

18. Torigoe AMS, Quagliato EMAB, Torigoe M, Carvalho KMM. Normatização do eletrorretinograma por reversão alternada de padrões em voluntários normais. Arq Bras Oftalmol. 2003;66(4):505-14.

19. IBGE. Censo Demográfico 2000. Fundação Seade (Sistema Estadual de análise de Dados) [citado 2010 jan 29]. Disponível em: http://www.seade.gov.br/ produtos/idr/download/populacao.pdf

20. Oliveira F. Ser negro no Brasil: alcances e limites. Estud Av. 2004;18(50): 57-60.

21. Pereira JM, Mendieta L, Sacai PY, Salomão SR, Berezovsky A. Estudo normativo do eletrorretinograma de campo total em adultos jovens. Arq Bras Oftalmol. 2003;66(2):137-44

22. Punaro E, Berezovsky A, Salomão SR. Eletrorretinografia focal em adultos jovens normais. Arq Bras Oftalmol. 2005;68(6):747-51.

23. Salomão SR, Ejzenbaum F, Berezovsky A, Sacai PY, Pereira JM. Age norms for monocular grating acuity measured by sweep-VEP in the first three years of age. Arq Bras Oftalmol. 2008;71(4):475-9.

24. Munhoz JS, Salomão SR, Berezovsky A, Sacai PY. Padronização normativa de eletro-oculografia em adultos. Arq Bras Oftalmol. 2004;67(2):207-10.

25. Paranhos FRL, Paranhos Júnior A, Nehemy MB. Eletrorretinograma: considerações a respeito dos limites de normalidade e comparação entre valores normais de dois diferentes laboratórios. Arq Bras Oftalmol. 2002;65(2):213-6.

26. Paranhos FRL, Ávila MP, Paranhos A, Cialdini AP. Estudo estatístico de valores normais do eletrorretinograma: contribuição à padronização do exame. Arq Bras Oftalmol. 1997;60(3):278-84. 